\title{
Radiation of anginal pain to the legs
}

\author{
MILTIADES T KOLETTIS, CHARALAMBOS K KALOGEROPOULOS, \\ GEORGE C TZANNETIS, SPYROS K VITAKIS, PETROS P XAPLANTERIS, \\ IOANNIS A NOVAS
}

From the Department of Cardiology, First Social Security Hospital, Athens, Greece

SUMMARY Pain radiated from the chest to one or both legs (17 cases), or from the legs to the chest (two cases) in 19 patients with angina or acute myocardial infarction. The leg pain was assumed to be related to the angina pectoris when both were of a similar character and occurred together, when the leg pain occurred at rest, and when there were normal peripheral pulses in the leg. Pain was felt in the left leg by 10 patients, the right leg by two patients, and in both legs by seven. Three patients experienced pain in the thigh(s), six in the shin(s), and 10 had pain in both. In six patients the pain extended down to the inner two to four toes. The pain was always felt in front of the legs. This distribution accords with the suggestion that some sensory cardiac nerve fibres occur in the lumbar sympathetic ganglia and that pain is projected into the corresponding dermatomes.

Despite improved diagnostic techniques, a history of angina is important in the diagnosis of ischaemic heart disease. ${ }^{1-3} \mathrm{~A}$ knowledge of the variations of anginal pain, including uncommon ones, will avoid errors in the diagnosis of a common disease.

We noticed that angina can extend to the legs in some patients with ischaemic heart disease.

\section{Patients and methods}

In 19 patients with ischaemic heart disease (16 men and three women, aged 27-73) angina was felt not only in the chest but also in one or both legs. We assumed that the pain in the legs was an extension of the cardiac pain when $(a)$ it was associated with chest pain due to typical angina or acute myocardial infarction or both; $(b)$ the diagnosis of angina was supported by electrocardiographic criteria at rest or during an exercise test or both; $(c)$ there was pain in one or both legs which resembled the chest pain and occurred at the same time; $(d)$ rest angina or acute myocardial infarction or both occurred with similar extension of the chest pain to the legs at some stage of the disease; and (e) normal arterial pulses were present in the legs. The last two criteria were included to exclude coincidental intermittent claudication occurring with anginal chest pain during effort.

Requests for reprints to Dr Miltiades Kolettis, 59 Ipsilantou Street, 11521 Athens, Greece.

Accepted for publication 3 September 1985

\section{Results}

All 19 patients had typical ischaemic chest pain. In 15 patients there was effort angina with chest pain of typical quality and distribution, and in 13 of these there were electrocardiographic changes indicating ischaemia during rest or during an exercise test or both. In eight of these patients coronary angiograms showed important coronary stenosis. The two patients with a normal resting electrocardiogram without (one case) or with doubtful (one case) exercise test both had positive coronary angiograms. All 15 patients also felt pain in one or both legs which passed off a few minutes after stopping physical effort or after taking sublingual glyceryl trinitrate or after both. Chest pain and leg pain or numbness resolved simultaneously. At some time these patients had also had chest pain at rest with radiation of pain to the legs. Seven patients had unstable angina and others had sporadic attacks of rest angina which occurred spontaneously (six cases) or during paroxysmal supraventricular tachycardia (one case) or during an attack of acute myocardial infarction (six cases).

The remaining four patients had no effort angina. One of them was a 27 year old man who had a constrictive pain across the chest, which radiated to both arms and the jaw; at the same time he had similar pain along the front of the right thigh and shin. Pain coincided with attacks of paroxysmal supraventricular tachycardia and was relieved immedi- 
Table Angiographic findings in patients with radiation of angina to the legs

\begin{tabular}{ll}
\hline & No of patients \\
\hline Coronary angiography: & \\
Three vessel disease & 4 \\
Two vessel disease & 4 \\
One vessel disease (proximal LAD) & 1 \\
Aortography: & 8 \\
Normal vessels & 1 \\
Minor stenosis in the femoral artery & 1 \\
\hline
\end{tabular}

$\mathrm{LAD}$, left anterior descending.

ately the tachycardia ended. The second patient was a 42 year old man who had severe constrictive pain in the upper surface and three or four inner toes of the right foot, which spread up to the right inguinal area and hypogastrium, and then extended widely across the chest. The next day the pain recurred but this time it started in both feet. The electrocardiogram showed $\mathrm{T}$ wave inversion in the inferior leads. The next morning the electrocardiogram showed a typical Wolff-Parkinson-White pattern which has persisted and has masked the evolution of other electrocardiographic changes. Estimation of cardiac enzymes suggested an evolving acute myocardial infarction. The third patient had had two episodes of acute myocardial ischaemia at rest six years before and an acute myocardial infarction one month before admission; during all three attacks there was extension of the chest pain to the left thigh. The fourth and last patient had had an inferior wall infarction in 1982 and five attacks of typical though prolonged angina pectoris occurring at rest during the past three months. Both during acute myocardial infarction and rest angina, the pain was felt in the left shin from below the knee to the four innermost toes.

Ten of the 19 patients had coronary angiography, and in all it showed important coronary artery disease (Table).

Pain spread to the left leg in 10 patients, the right leg in two patients, and to both legs in seven cases. In all cases it affected the front of the legs. The pain affected the thigh(s) in three patients; the shins in six; both in ten, and in one patient it was more widely distributed. In two cases pain spread from the legs to the chest. In six it extended to the feet and to two to four of the inner toes.

In all 19 cases the peripheral pulses in the legs were normal. Aortography was carried out in nine patients (Table) and was normal in eight, while in one there was slight narrowing $(40 \%)$ of the right deep femoral artery. The arteries of the left leg in which the pain was felt were normal.

\section{Discussion}

The chest is the classic site of angina and such pain often extends to the upper half of the body, particularly the arms and forearms, the neck, the jaw, and the interscapular areas. ${ }^{145}$ Its extension to the lower half of the body, particularly to the legs, is not so well recognised. Textbooks ${ }^{5-7}$ and review articles ${ }^{28}$ indicate that angina rarely if ever extends below the epigastrium. Extension of angina to the legs in two out of 160 patients was reported by McKenzie in $1923 .{ }^{9}$ We believe that this radiation of the pain is considerably more common than the few reported cases indicate.

We report 19 cases of chest pain due to angina or acute myocardial infarction or both in which pain was also felt in the legs. The possibility that pain in the legs was caused by coexistent intermittent claudication was excluded by the presence of normal peripheral arterial pulses in the legs and the occurrence of the pain in the legs during rest angina or acute myocardial infarction.

In these patients angina spread to the thighs, the shins, or both and in some cases also extended to the feet including the inner two to four toes, but not the small toe. The pain was more common in the left leg. Both legs were affected in some cases. In general angina radiated from the chest to the legs but in two patients it spread from the legs to the chest. The pain was felt in the front of the legs whereas the pain of intermittent claudication is felt mainly in the backs of the legs. Anterior tibial compartment syndrome, which is caused by muscle oedema and tension of the fascia tibialis, produces pain resembling angina at the front and sides of the shin ${ }^{1011}$ and the occurrence of pain in the thigh or foot excludes this syndrome. Even when the angina is limited to the tibial area, anterior tibial compartment syndrome, which predominantly affects very young people, is highly unlikely to be found coincidentally in a patient with typical angina pectoris and such a diagnosis would certainly be excluded when there is also angina pectoris at rest, as was the case in all of our patients.

The mechanism of extension of angina to the legs could be the result of some sensory cardiac nerves being present in the lower sympathetic ganglia, rather than being restricted to the seventh and eighth cervical and the first four or five thoracic sympathetic ganglia, ${ }^{4612}$ and the consequent projection of angina to the corresponding dermatomes. The extension of the pain to the front of the legs and the four inner toes is compatible with the presence of sensory cardiac nervous fibres in the lumbar sympathetic ganglia, whereas the backs of the legs and the small toe are innervated by the sacral sympathetic ganglia. ${ }^{12}$ 


\section{References}

1 Andrus EC. Diagnosis of angina pectoris. Circulation 1960; 22: 979-85.

2 Sampson JJ, Cheitlin MD. Pathophysiology and differential diagnosis of cardiac pain. Prog Cardiovasc Dis 1971; 13: 507-31.

3 Detry JMR, Kapita BM, Cosyns J, Sottiaux B, Brasseur LA, Rousseau MF. Diagnostic value of history and maximal exercise electrocardiography in men and women suspected of coronary heart disease. Circulation 1977; 56: 756-61.

4 Lewis T. Pain in muscular ischaemia; its relation to anginal pain. Arch Intern Med 1932; 49: 713-27.

5 Hurst JW. The heart. 4th ed. New York: McGraw-Hill, 1978: 28 and 1066-70.

6 Friedberg CK. Diseases of the heart. 3rd ed. Philadel- phia and London: Saunders, 1966: 706-26.

7 Braunwald E. Heart disease. A textbook of cardiovascular medicine. Philadelphia: Saunders, 1980: 1388-9.

8 Dressler W. Clinical aids in cardiac diagnosis. New York: Grune and Stratton, 1970: 14-49.

9 McKenzie J. Angina pectoris. Oxford Medical Publications. London: Frowde, Hodder and Stoughton, 1923.

10 Kennelly BM, Blumberg L. Bilateral anterior tibial claudication. Report of two cases in which the patients were cured by bilateral fasciotomy. $\mathfrak{F} A M A$ 1968; 203: 487-91.

11 Jantet $G$. The anterior tibial compartment syndrome. In: Taylor S, ed. Recent advances in surgery, 9. Edinburgh, London, New York: Churchill Livingstone, 1977: 361-9.

12 Sobotta J. Atlas der Anatomie des Menschen (Greek translation). München, Berlin, Wien: Urban and Schwarzenberg, 1978: 78-9. 